ISSN (O) 2589-8779

(P) 2589-8760

CMRO 04 (05), 950-960 (2021)

RESEARCH ARTICLE

\title{
Real-world clinical experience of extended half-life recombinant factor VIII Fc fusion protein (rFVIIIFc) in comparison to conventional factor products in patients with severe hemophilia $A$
}

\author{
Georg Goldmann $^{1}$ | Natascha Marquardt ${ }^{1}$ | Silvia Horneff ${ }^{1}$ | Claudia Klein ${ }^{1}$ \\ | Thilo Albert ${ }^{1}$ | Johannes Oldenburg ${ }^{1^{*}}$
}

\footnotetext{
${ }^{1}$ Institute of Experimental Hematology and Transfusion Medicine, University Clinic Bonn, Bonn, Germany
}

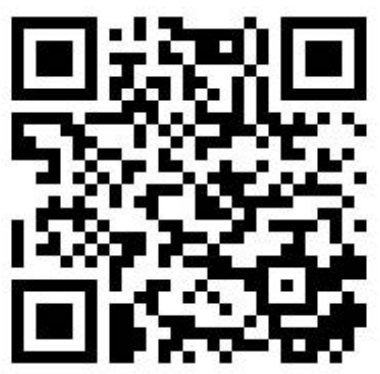

\begin{abstract}
Introduction: The recombinant factor FVIII Fc fusion protein (rFVIIIFc) is a first-in-class extended half-life FVIII product to treat patients with hemophilia A. The safety, efficacy and prolonged half-life of rFVIIIFc was demonstrated in the phase 3 studies A-LONG, Kids ALONG and the extension study ASPIRE. Despite robust efficacy and safety data of rFVIIIFc therapy from clinical trials, evidence on the effectiveness of rFVIIIFc use in real-world remains scarce. Our analysis aimed at investigating the effectiveness of prophylactic rFVIIIFc treatment in routine clinical use in Germany.
\end{abstract}

Material and Methods: Twenty-seven patients with severe hemophilia A, who switched from prophylaxis with conventional recombinant factor VIII (rFVIII) products to rFVIIIFc, were included. Annualized bleeding rates, factor consumption, number of injections and adherence to prophylaxis were compared. The retrospective period prior switching to rFVIIIFc was three years, while the mean follow-up period after switching to rFVIIIFc was 24.9 months.

Results: Switching to rFVIIIFc led to a $33.7 \%$ reduction in mean annualized number of injections and a $18.3 \%$ reduction in mean annualized factor consumption while maintaining low bleeding rates. The mean annualized bleeding rate (ABR) was 2.5 and 1.7 for rFVIII and rFVIIIFc, respectively. The adherence improved from $87 \%$ to $94 \%$. During the follow-up period eleven surgeries were performed; all with a hemostatic response rated as excellent. No FVIII inhibitor formation after switching to rFVIIIFc has been detected.

Conclusion: Real-world treatment with rFVIIIFc was associated with substantial reductions in consumption and injection frequenies while maintaining low bleeding rates supporting safety and efficacy data from clinical trials.

Keywords: severe hemophilia A, recombinant factor VIII Fc fusion protein, extended half-life, prophylaxis, real-world clinical experience.

Copyright : (C) 2021 The Authors. Published by Publisher. This is an open access article under the CC BY-NC-ND license (https://creativecommons.org/licenses/by-nc-nd/4.0/). 


\section{1 | INTRODUCTION}

$\mathrm{H}$ emophilia $\mathrm{A}$ is a rare $\mathrm{X}$-linked genetic bleeding disorder caused by coagulation factor VIII (FVIII) deficiency resulting in impaired hemostasis and prolonged bleeding episodes. Intra-articular and intramuscular bleeding is the major clinical manifestation of the disease. Recurrent hemarthrosis and soft-tissue hematomas are the major cause of severe arthropathy, joint contractures, and pseudotumors, which may cause chronic pain, limit physical mobility, and overall impair the quality of life $[(1,2)$.

Prophylactic replacement therapy of FVIII to prevent bleeding is the standard of care (3-5).

Conventional FVIII replacement products are either plasma-derived or recombinant with a standard half-life (SHL) of approximately 8-12 hours necessitating frequent intravenous injections of at least three times per week in most patients to maintain plasma levels above $1 \mathrm{IU} / \mathrm{dL}$ (6). The burden of frequent injections can lead to patients' avoidance or delay in starting prophylaxis consequently increasing the risk of morbidities, especially hemophilic arthropathies (7). It can also impact adherence to the prescribed regimen as expressed by delayed administrations or skipped infusions (8).

Several new treatment options have emerged in recent years. Extending FVIII half-life is one key strategy to primarily increase bleed protection but also to lessen treatment burden (9). The extended half-life (EHL) FVIII concentrate Efmoroctocog alfa (rFVIIIFc) was approved as a first-in-class recombinant FVIII (rFVIII) Fc-fusion protein with EHL for the treatment of patients with hemophilia A (PwHA) of all age groups in Europe in 2015 (10). It is composed of a single molecule of B-domain-deleted rFVIII covalently linked to the Fc domain of human immunoglobulin G1 (IgG1) (11). The fusion to the IgG1 Fc domain prolongs the half-life through a mechanism involving the endogenous IgG recycling pathway by interaction with the neonatal Fc receptor (FcRn) (12). rFVIIIFc is expressed in human embryonic kidney (HEK) $293 \mathrm{H}$ cells without any addition of exogenous human- or animal-derived protein in the cell culture process, purification or final formulation (10). Comparative pharmacokinetic data for
rFVIIIFc and conventional rFVIII showed a similar incremenmtal recovery and volume of distribution, but a reduced clearance of $\mathrm{rFVIIIFc}(13,14)$. This reduction in clearance leads to a median 1.5 -fold prolongation in elimination half-life of rFVIIIFc (14).

The efficacy and safety of rFVIIIFc have been demonstrated in two pivotal phase III clinical trials: A-LONG in adults and adolescents aged $\geq 12$ years (NCT01181128) (14) and Kids A-LONG in children aged $<12$ years (NCT01458106) (15). Efficacy and safety data from the rFVIIIFc extension trial, ASPIRE (NCT01454739), confirmed long-term safety of rFVIIIFc and the maintenance of a low annualized bleeding rate $(\mathrm{ABR})(16,17)$.

Pharmacokinetic simulations demonstrate that the lower clearance of rFVIIIFc compared to standard half-life FVIII has the potential of improved bleed prevention without increasing overall factor consumption (18). Higher trough levels of plasma FVIII can be achieved to enable patients to a more active life and cause higher protection. Effective prevention of bleeding episodes may be beneficial to longterm joint health, and a trend towards continued improvement in joint health during the ASPIRE extension study was observed (19). Despite a recent uptake in published real-world studies for the use of EHL factor concentrates, data on the effectiveness of rFVIIIFc in clinical practice overall remains scarce. Here, we report our real-world clinical experience on patients with severe hemophilia A after switching from prophylactic treatment with $\mathrm{rFVIII}$ to $\mathrm{rFVIIIFc}$ with a retrospective period of 36 months and a mean follow-up of 24.9 months.

Supplementary information The online version of this article (https://doi.org/10.15520/jcmro.v4i05.42 2) contains supplementary material, which is available to authorized users.

\section{Corresponding Author: Johannes Oldenburg}

Institute of Experimental Hematology and Transfusion Medicine, University Clinic Bonn, Bonn, Germany Institute of Experimental Hematology and Transfusion Medicine, University Clinic Bonn, Bonn, Germany 


\section{I MATERIALS AND METHODS}

\section{Study population and data collection}

Previously treated male patients with severe hemophilia A, who switched from prophylactic treatment with conventional recombinant factor products to rFVIIIFc (efmoroctocog alfa, Swedish Orphan Biovitrum AB) prophylaxis in 2016 or 2017, were retrospectively included in the study. Twentysix patients had a previous prophylaxis with octocog alfa and one patient received simoctocog alfa prophylaxis prior to switching to rFVIIIFC. All data are clinical routine data and were collected at the Institute of Experimental Hematology and Transfusion Medicine, University Clinic Bonn, Bonn, Germany. Patient characteristics (age, weight, F8 genotype, presence of infectious diseases or arthropathies), data of rFVIII and rFVIIIFc treatment (prescribed injection intervals, dose per injection, and start date of rFVIIIFc treatment) as well as the number of bleeds (overall, spontaneous, traumatic, joint, spontaneous joint) were collected. The initial switch from pre-medication to Elocta was performed according to the SPC label. If necessary during follow-up patients' treatment was adapted according to individual needs. Data cut-off for rFVIIIFc treatment was on July 31, 2018. Adherence to prophylaxis was evaluated using patient diaries. All surgeries were planned according to localisation, type and complexity and included a substitution plan with the respective factor concentrate as well as the time of injections. For major surgeries the recommendation of the initial dose is $80-100 \mathrm{IU} / \mathrm{kg}$ and 50-80 IU/kg in children and adults, respectively. For minor surgeries the recommendation of the initial dose is $50-100 \mathrm{IU} / \mathrm{kg}$ and $25-40 \mathrm{IU} / \mathrm{kg}$ in children and adults, respectively [20]. To aim for avoidance of wound healing disturbances and postoperative hemorrhages the factor trough level for major surgeries should be in normal range for 710 days post-surgery to continuously support the wound healing processes. All patients had a first injection with factor concentrate the evening before surgery (preloading dose). On the day of surgery all patients received a loading dose and dependend of the time to surgery an additional factor concentrate injection immediately before the operation. Follow up injections were based according to the actual factor activity. Total number of injections and factor consumption was calculated peri-operatively for a maximum follow-up time of 10 days post-surgery.

For this retrospective analysis patients data were anonymized and aggregated.

\section{Statistical analysis}

For both FVIII and rFVIIIFc treatment, the annualized number of injections was calculated. The percent change in the annualized number of injections after the switch to rFVIIIFc was determined. In analogy, annualized factor consumption and percent change in annualized factor consumption was calculated.

Bleeding rates during FVIII prophylaxis were calculated for a time period of three years and converted to annualized bleeding rates (ABRs). For rFVIIIFc prophylaxis, ABRs were calculated based on the number of bleeds during the follow-up period since start of rFVIIIFc treatment until the date of evaluation. Analogously, ABRs were calculated separately for spontaneous bleeds (AsBR), traumatic bleeds (AtBR), joint bleeds (AjBR), and spontaneous joint bleeds (AsjBR).

Adherence to prophylactic treatment was determined as the percentage of actual injections of prescribed injections.

Descriptive statistics report numbers and percentages for categorical variables and mean and standard deviation (SD) for continuous variables. Wilcoxon signed-rank test was used for paired samples. $\mathrm{P}<0.05$ was considered statistically significant.

\section{3 | RESULTS}

\section{Patient characteristics}

In total, 27 previously treated patients with severe hemophilia A were included. Demographics and baseline characteristics are presented in Table1. The cohort comprised 24 adult and adolescent $(\geq 12$ years) and 3 pediatric patients. All patients were male with a mean age of 37 years. All patients had F8 gene mutations of which 21 patients $(77.8 \%)$ presented with a genotype which was associated 
Real-world clinical experience of extended half-life recombinant factor VIII Fc fusion protein (rFVIIIFc) in comparison to conventional factor products in patients with severe hemophilia $\mathrm{A}$

with an increased risk of inhibitor development, i.e. 14 intron 22 inversions, four stop mutations, two splice mutations in a conserved region and one small deletion. The remaining six patients $(22,2 \%)$ displayed mutations which were associatetd with a low risk of inhibitor development (three missense mutations, one duplication, one splice mutation in a non conserved region and one small deletion in a repetive adenine nucleotide region). Of all 27 patients four had a prior history of inhibitors to FVIII (two patients with an intron 22 inversion, one patient with a stop mutation and one patient with a splice mutation in a conserved region) with two testing positively for high-titer inhibitors ( $>5 \mathrm{BU})$ and two for low-titer inhibitors $(<5 \mathrm{BU})$, respectively.. Of note, none of them developed a recurrent inhibitor after switching to rFVIIIFc. Nine patients (33.3\%) had infectious diseases such as human immunodeficiency virus [HIV], hepatitis $\mathrm{C}$ virus [HCV], or both, and 13 patients $(48,1 \%)$ had at least one joint with arthropathies. Retrospective observational period with conventional rFVIII concentrate was 3 years for all patients. The mean follow-up period after switching to rFVIIIFc treatment was 24.9 months.

Prophylactic regimens and adherence

Switching to rFVIIIFc prophylaxis led to a $33.7 \%$ reduction of mean injection frequency compared to prior conventional rFVIII therapy $(121 \pm 30$ vs. 182 \pm 71 injections per year) corresponding to a mean reduction from 3.5 to 2.3 injections per week. In total, $22(81.5 \%)$ of the 27 patients were able to extend their injection interval Table 2 .

Of those, 12 patients reduced the interval from 3 to 2 injections/week, 6 patients from every other day (3.5 injections/week) to either 2 injections/week $(n=3)$, every 3 (2.3 injections/week) days $(n=1)$, or 3 injections/week $(n=2)$ and one patient from 4 injections/week to every other (3.5 injections/week) day. Three patients with daily injections were able to reduce the injection interval to either every 3 days $(n=1)$ or 3 injections per week $(n=2)$. In five patients, injection intervals did not change, two of those had already a low injection interval during conventional rFVIII therapy (one and two injections per week).

In the patient population of this study (26 of 27 patients with available data for both treatment regimens), mean adherence to rFVIII prophylaxis was

\begin{tabular}{|c|c|}
\hline All patients $(n=27)$ & \\
\hline \multicolumn{2}{|l|}{ Age, years } \\
\hline Mean \pm SD & $36.8 \pm 20.7$ \\
\hline Median (min, max) & $40.0(9.0 ; 81.0)$ \\
\hline \multicolumn{2}{|l|}{ Weight, kg } \\
\hline Mean \pm SD & $66.8 \pm 26.7$ \\
\hline Median (min, max) & $66.0(19.0 ; 120.0)$ \\
\hline \multicolumn{2}{|l|}{ Observation time, months } \\
\hline Mean \pm SD & $24.9 \pm 4.2$ \\
\hline Median (min, max) & $24.6(13.9 ; 29.6)$ \\
\hline Total Elocta units (IU) & $14,307,970$ \\
\hline \multicolumn{2}{|l|}{ Age Distribution ( $n$ ) } \\
\hline$<12$ years & 3 \\
\hline$\geq 12$ to $<18$ years & 5 \\
\hline 18 to $<60$ years & 15 \\
\hline$\geq 60$ years & 4 \\
\hline \multicolumn{2}{|l|}{ Infectious Diseases (n) } \\
\hline HIV and HCV & 4 \\
\hline HIV & 4 \\
\hline $\mathrm{HCV}$ & 1 \\
\hline \multicolumn{2}{|l|}{ Arthropathies* (n) } \\
\hline Ankle & 26 \\
\hline Knee Joint & 11 \\
\hline Hip Joint & 1 \\
\hline Cubital Joint & 10 \\
\hline
\end{tabular}

FIGURE 1: Table 1. Patient characteristics

$87 \pm 16 \%$ and after switching to $\mathrm{rFVIIIFc}$, adherence increased to $94 \pm 8 \%$.

\section{Factor consumption}

The mean annualized factor consumption of rFVIII was $295,461 \pm 130,914 \mathrm{IU}$ and was reduced by $18.3 \%$ to $241,509 \pm 78,644$ IU with rFVIIIFc, corresponding to $4,896 \pm 2,167 \mathrm{IU} / \mathrm{kg} \mathrm{BW} /$ year and $3,975 \pm 1,382 \mathrm{IU} / \mathrm{kg} \mathrm{BW} /$ year, respectively. In order to improve bleeding protection, factor reduction did not apply for eight patients. Two patients increased their consumption $(156,000$ to $286,000 \mathrm{IU} /$ year and 


\begin{tabular}{|c|c|c|c|c|c|c|c|}
\hline rFVIII & Daily & $4 x / w k$ & EOD* & $3 x / w k$ & Q3 $D^{* *}$ & $2 x / w k$ & $1 \mathrm{x} / \mathrm{wk}$ \\
\hline Daily & & & & 2 & 1 & & \\
\hline $4 x / w k$ & & & 1 & & & & \\
\hline EOD* & & & 1 & 2 & 1 & 3 & \\
\hline $3 x / w k$ & & & & 2 & & 12 & \\
\hline \multicolumn{8}{|l|}{ Q3D** } \\
\hline $2 x / w k$ & & & & & & 1 & \\
\hline $1 x / w k$ & & & & & & & 1 \\
\hline
\end{tabular}

* every other day

${ }^{* *}$ every three days

In the patient population of this study ( 26 of 27 patients with available data for both treatment regimens), mean adherence to rFVIII prophylaxis was $87 \pm 16 \%$ and after switching to rFVIIIFc, adherence increased to $94 \pm 8 \%$.

FIGURE 2: Changes of prophylaxis infusion regimens afterswitch from conventional rFVIII to rFVIIIFc concentrate. Values represent npatients. Greyed boxes indicate where dosages remained unchanged.

234,000 to $273,000 \mathrm{IU} /$ year, respectively). The mean weekly dose of rFVIIIFc was $76.5 \pm 27 \mathrm{IU} / \mathrm{kg} / \mathrm{wk}$ and the mean weekly dose of rFVIII was $94.1 \pm 42$ $\mathrm{IU} / \mathrm{kg} / \mathrm{wk}$.

Intra-patient comparison of weekly factor consumption is shown in Figure 1.

\section{Annualized bleeding rates}

The mean ABR and mean joint ABR (AjBR) were $2.5 \pm 3.1$ and $0.7 \pm 1.5$ for rFVIII and $1.7 \pm$ 3.7 and $0.3 \pm 0.6$ for rFVIIIFc, respectively. Mean spontaneous $\mathrm{ABR}$ (AsBR) and mean spontaneous joint ABR (AsjBR) were $0.4 \pm 0.7$ and $0.2 \pm 0.4$ for conventional rFVIII and $0.2 \pm 0.5$ and $0.2 \pm 0.4$ for rFVIIIFc, respectively. Bleeding data prior to, and after initiating treatment with $\mathrm{rFVIIIFc}$ are shown in Figure 2.

There were no serious spontaneous bleeding episodes with rFVIIIFc prophylaxis during the follow-up period of this study, which would have required long-term inpatient or outpatient treatment.

\section{Perioperative management}

Hemostatic efficacy was rated as excellent during all surgical procedures as defined by Srivastava et al. (20) [20]. Mean consumption in the postoperative setting per patient as well as treatment days are shown in Table 3. In total, eleven surgeries had been conducted in nine patients with severe hemophilia A, after switching to rFVIIIFc. Of five major surgeries, four were orthopaedic. Six interventions were considered minor surgeries. All patients adhered to their prescribed treatment regimen prior to surgery. The evening before surgery, a mean preloading dose of $38( \pm 7)$ and $34( \pm 13) \mathrm{IU} / \mathrm{kg}$ rFVIIIFc was given in major and minor surgeries, respectively. Prior to surgery, an additional dose of $56( \pm 30) \mathrm{IU} / \mathrm{kg}$ in major and $49( \pm 22) \mathrm{IU} / \mathrm{kg}$ in minor surgeries was administered Table 4 .

\section{4 | DISCUSSION}

Successful long-term outcome in PwHA consists of an efficient prophylaxis to reduce the occurrence of bleeding episodes and to maintain joint health. Despite widespread availability of safe and effective replacement therapies, PwHA remain to be exposed to a significant treatment burden of frequent intravenous injections, while still experiencing breakthrough bleedings, and progressive joint disease over a lifetime $(9,21-24)$. Novel therapeutic approaches have been developed to overcome these challenges including Efmoroctocog alfa (rFVIIIFc). Attachment to the IgG1 Fc domain permits binding to the FcRn, which is responsible for protection of IgG from lysosomal degradation and facilitates its recycling (12). As a result, rFVIIIFc shows prolonged clearancerelated pharmacokinetics with the potential to improve bleed prevention without increasing the over- 
Real-world clinical experience of extended half-life recombinant factor VIII Fc fusion protein (rFVIIIFc) in comparison to conventional factor products in patients with severe hemophilia A

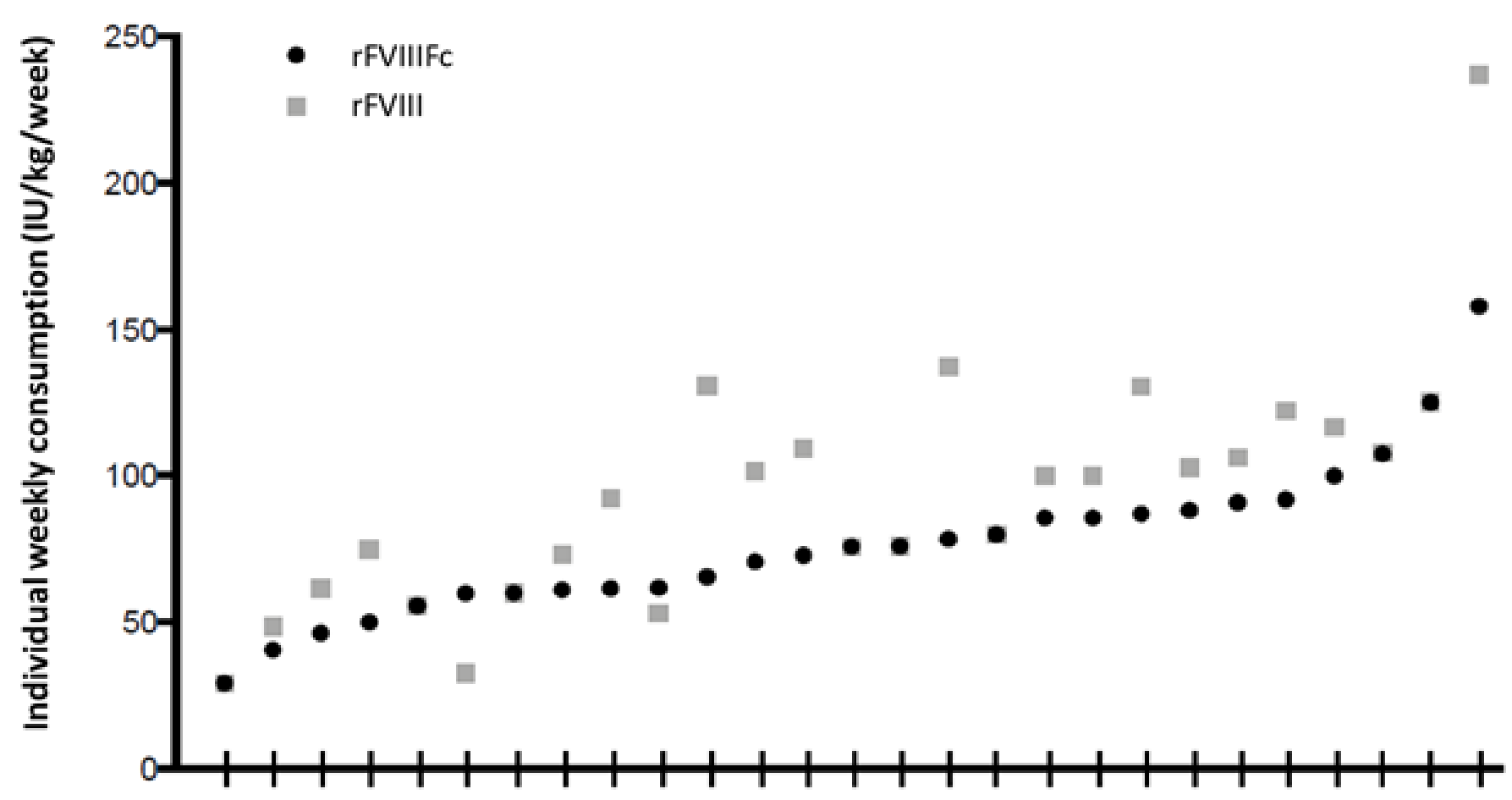

Individual Patients

FIGURE 3: Intra-patient dosingfor prophylaxis with rFVIIIFc and previous rFVIII product. X-axis showsindividual patient data. Dosing for each patient on rFVIIIFc is plotted inblack; dosing with previous rFVIII product is plotted in grey.

all factor consumption and concurrently to reduce treatment burden with less frequent injections (13, 14, 18).

In the present study, the real-world usage and potential therapeutic benefits of long-acting rFVIIIFc were analyzed in 27 patients with severe hemophilia A previously treated with conventional recombinant factor products. The mean follow-up period after switching to rFVIIIFc was 24.9 months. While randomized clinical trials remain the gold standard to establish efficacy and safety of new therapeutic agents, data from clinical routine produce evidence of therapeutic effectiveness in real-world practice settings. Extensive analyses from clinical trials have been published (13-17, 25-31), but uncertainties prevail among physicians despite of a recent uptake in published real-world evidence (32-38). Especially, the inhibitor risk of patients with a prior history of inhibitors is an issue of debate since those patients are excluded from clinical trials. In our study four patients with a prior history of inhibitors (two with high-titer inhibitors, two with low-titer inhibitors) against FVIII switched to rFVIIIFc and none of those patients developed inhibitors. These data are consistent with an analysis of 36 patients pooled from the phase 4 observational study programs of PREVENT and A-SURE, respectively (39). Furthermore, a recently published Italian single-center study corroborates these findings for the use of rFVIIIFc in clinical routine (37). In our study, overall, the weekly dose of rFVIIIFc was lower compared to that of previous SHL FVIII concentrate. The observed reduction of $18.3 \%$ corresponds to $19 \%$ reported in a Canadian analysis (40) and $17-26 \%$ according to a meta-analysis (41). In the aforementioned single center study conducted in Italy Tagliaferri et al. report a $\sim 12 \%$ reduction in weekly dose (37). Importantly, the reduction in factor consumption did not adversely affect bleed protection. Iorio et al. detected significantly lower ABRs on rFVIIIFc compared to 


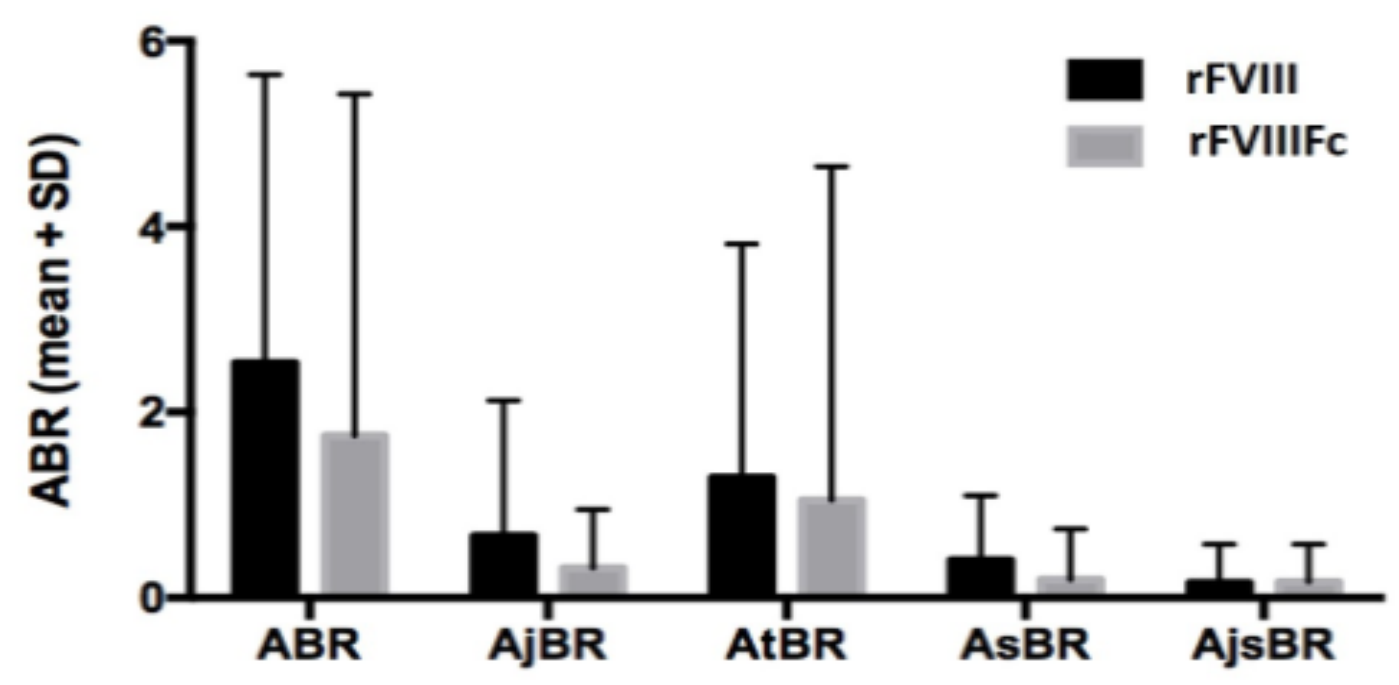

(A)

(B)

\begin{tabular}{|l|c|c|c|c|c|c|c|c|c|c|}
\hline & \multicolumn{2}{|c|}{ ABR $^{*}$} & \multicolumn{2}{c|}{ AjBR** $^{* *}$} & \multicolumn{2}{c|}{ AtBR $^{* * *}$} & \multicolumn{3}{c|}{ AsBR $^{* * *}$} & \multicolumn{2}{c|}{ AsjBR $^{* *}$} \\
\hline & rFVIII & rFVIIIFC & rFVIII & rFVIIIFC & rFVIII & rFVIIIFC & rFVIII & rFVIIIFC & rFVIII & rFVIIIFC \\
\hline Median & 1.7 & 0.5 & 0 & 0 & 0.3 & 0 & 0 & 0 & 0 & 0 \\
\hline (min; max) & $0.0 ; 11.3$ & $0.0 ; 18.8$ & $0.0 ; 7.3$ & $0.0 ; 2.6$ & $0.0 ; 11.0$ & $0.0 ; 18.8$ & $0.0 ; 2.7$ & $0.0 ; 2.6$ & $0.0 ; 2.0$ & $0.0 ; 1.6$ \\
\hline
\end{tabular}

* Annualized bleeding rate

$* *$ Annualized joint bleeding rate

${ }_{* * *}$ Annualized traumatic bleeding rate

$* * * *$ Annualized spontaneaous bledding rate

\# Annualized spontenaous joint bleeding rate

FIGURE 4: Mean annualizedbleeding rates (ABRs) in patients who received $\mathrm{FFVIIIFc} \mathrm{prophylaxis} \mathrm{compared}$ toprophylaxis on prior $r F V I I I$ product $(A)$. Median ABR values and minumum-to-maximumranges are presented below (B).

\begin{tabular}{|l|l|l|l|l|l|l|l|}
\hline & \multicolumn{2}{|l|}{ Major surgeries (N=5) } & \multicolumn{2}{l|}{ Minor surgeries (N=6) } & \multicolumn{2}{l|}{ All surgeries (N=11) } \\
\hline & Mean & (Min-Max) & Mean & (Min-Max) & Mean & (Min-Max) \\
\hline Number of injections & 28 & $(12-61)$ & 5 & $(1-30)$ & 18 & $(2-61)$ \\
\hline Treatment duration (days) & 25 & $(9-57)$ & 4 & $(1-23)$ & 15.5 & $(2-57)$ \\
\hline Total dose (IU/kg)* & 967 & $(566-2,593)$ & 227 & $(57-1,250)$ & 660 & $(57-2,593)$ \\
\hline
\end{tabular}

*Total consumption was calculated for the first 10 treatment days.

FIGURE 5: Post surgerytreatment with rFVIIIFc. Number of injections, treatment durations andtotal consumption are shown for major, minor, all surgeries. Referring valuesare provided as mean and as minimum-to-maximum range, repectively. 
Real-world clinical experience of extended half-life recombinant factor VIII Fc fusion protein (rFVIIIFc) in comparison to conventional factor products in patients with severe hemophilia A

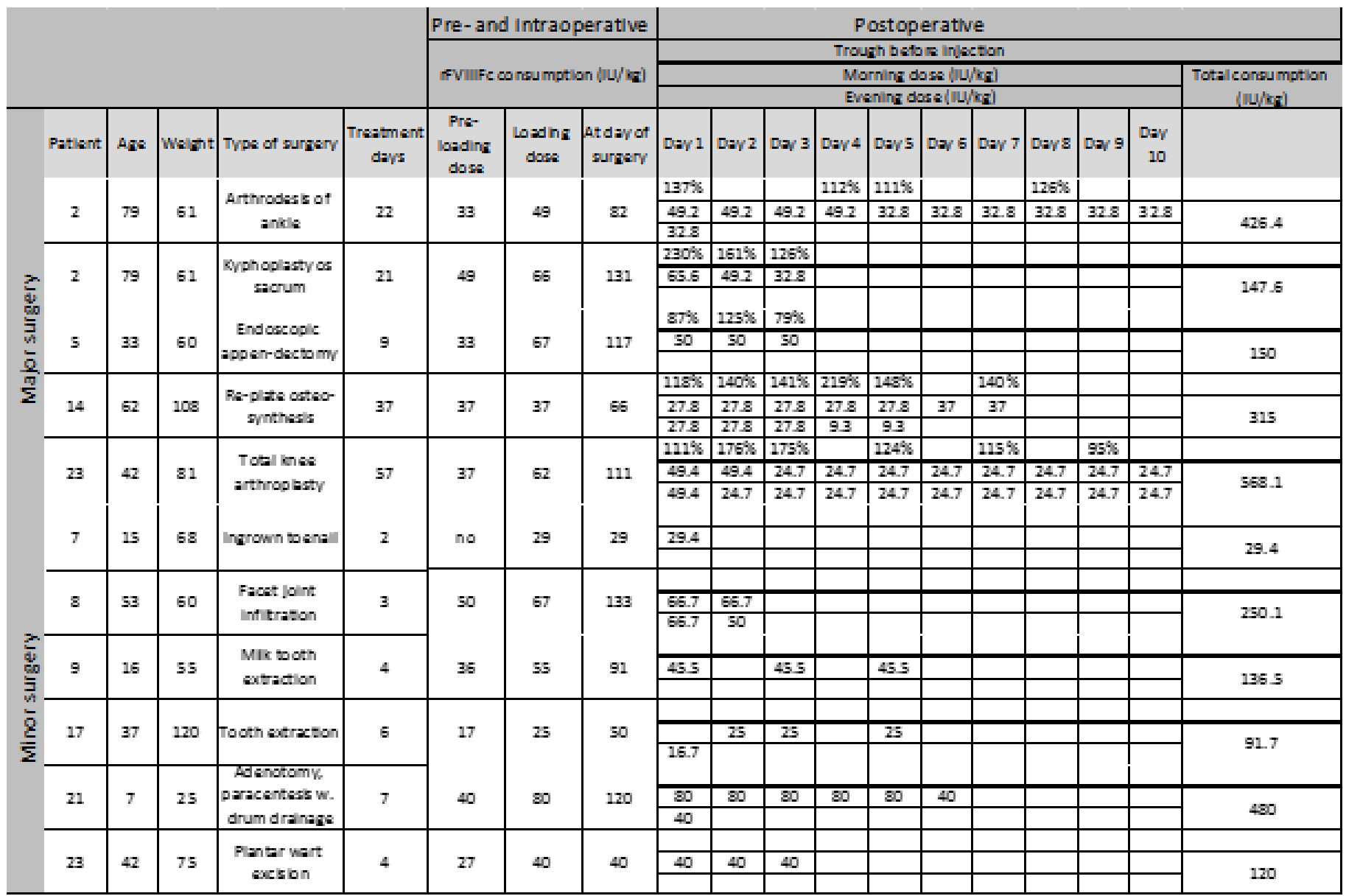

FIGURE 6: Overview of the elevensurgeries conducted in nine patients with severe hemophilia A post-switch to rFVIIIFc: patients' age andweight, intervention type, pre-, peri- and post-surgery treatment with rFVIIIFc,rFVIIIFc factor consumption.

standard rFVIII, while ABRs remained stable in our and the Italian study.

The improved prevention of bleeding episodes, especially in joints $(70 \%$ of patients with zero AjBR within 2 years observation time), might be likely beneficial to long-term joint health.

The reduced clearance of rFVIIIFc allows for a high degree of treatment personalization. PwHA may be enabled to reduce annualized factor consumption and mean dosing frequency to lift the burden of frequent injections. Conversely, an alternative treatment strategy can result in treatment intensification to improve bleed protection and joint health by raising the factor trough levels from $1 \%$ to $3-5 \%$ or even higher. This assumption is supported by recently published efficacy data on another EHL, rurioctocog alfa pegol, by Klamroth et al. (42). In concordance with this uplift of target trough levels concept PwHA may be switched from SHL to EHL in a 1:1 manner with respect to injection dose and injection frequency (18). Revised treatment strategies as such are increasingly being supported by national and international treatment recommendations and guidelines, that advocate for target trough levels of $1-3 \%$ or more recently even $3-5 \%(20,43-45)$. Observations from clinical practice according to which PwHA dosed to trough levels of $1 \%$ still display a considerable risk for developing hemophilic arthropathies are dissatisfactory (21). This and the introduction of EHL factor concentrates such as rFVIIIFc advocate for a more progressive preservation of joint health. Doing so, the otherwise inevitable detrimental triad of recurrent joint bleeds, chronic synovitis and hemophilic arthropathy might be almost prevented.

Adherence to FVIII prophylaxis was generally high in the patient population of this study. However, after 
switching to $\mathrm{rFVIIIFc}$, adherence further improved significantly from $87 \%$ to $94 \%$. The data are in accordance with a review of Ingersoll and Cohen who described that treatment burden and regimen complexity are likely determinants of adherence. Less complex regimens with fewer doses, by contrast, were shown to promote adherence (7). In this regard the benefits of higher trough levels for joint health at equal dosing intervals should be carefully weighed against the possible risk of lower treatment adherence. Hence, the treatment strategy of choice needs to fit the individual needs of the respective patient (46).

In addition to bleeding prevention in the daily lives of PwHA, surgical procedures require an optimal therapeutic management for adequate hemostasis. Eleven surgeries were performed with rFVIIIFc within this study. Importantly, rFVIIIFc was shown to be safe and effective in the perioperative setting, and hemostasis management was excellent in both major and minor surgeries.

Limitations of this study are the relatively small number of patients and the single center design. Furthermore, our hemophilia A cohort exclusively consists of patients who had switched to rFVIIIFc from a prior replacement product. Despite the potential limitations of our analyses, however, the results are consistent with published data on rFVIIIFc and add to the body of real-world evidence regarding the therapeutic effectiveness and safety of rFVIIIFc in PwHA previously treated with conventional factor concentrates.

\section{5 | CONCLUSION}

This is the first study to provide real-world evidence on personalized prophylaxis in a German cohort of PwHA being switched from conventional SHL factor concentrates to rFVIIIFc. Data were in line with previously published pivotal $\mathrm{rFVIIIFc}$ studies.

Prophylaxis with rFVIIIFc was efficacious in all patients and generally well tolerated. There was no inhibitor development, even in patients with inhibitor history. rFVIIIFc was associated with low overall and joint bleeds as well as significantly improved treatment adherence. Perioperative management was performed according to guidelines and hemostatic efficacy was rated as excellent.

Our findings suggest that rFVIIIFc can improve effectiveness compared to conventional rFVIII concentrates in real-world clinical settings.

\section{Declaration of interest}

Johannes Oldenburg: Honoraria; fees for speaking, consulting or symposium/congress attendance; or research funding: Bayer, Biogen Idec, Biotest, Chugai, CSL Behring, Grifols, Novo Nordisk, Octapharma, Pfizer, Roche, Shire Swedish Orphan Biovitrum.

Georg Goldmann: Honoraria; fees for speaking, consulting or symposium/congress attendance; or research funding: Bayer, Biotest, Chugai, CSL Behring, Novo Nordisk, Octapharma, Pfizer, Roche, Swedish Orphan Biovitrum, Takeda.

Natascha Marquardt: Honoraria; fees for speaking, consulting or symposium/congress attendance; Bayer, Chugai, CSL Behring, Novo Nordisk, Octapharma, Pfizer, Roche, Shire and Swedish Orphan Biovitrum.

Claudia Klein: Not applicable

Silvia Horneff: Honoraria; fees for speaking, consulting or symposium/congress attendance; Roche, Octapharma, Bayer, Novo Nordisk, Swedish Orphan Biovitrum

Thilo Albert: Funds and/or reimbursement for attending symposia, congresses and/or investigator meetings from Baxter/Baxalta/Shire/Takeda, Bayer, Biogen Idec, Biotest, Chugai, CSL-Behring, Grifols, Novo Nordisk, Octapharma, Roche, Swedish Orphan Biovitrum, and Pfizer.

\section{Author contribution}

Georg Goldmann: Conceptualization, Methodology, Investigation, Writing- Original draft preparation

Natascha Marquardt: Investigation, Writing- Reviewing and Editing

Silvia Horneff: Data curation, Writing- Reviewing and Editing,

Claudia Klein: Investigation, Writing- Reviewing and Editing

Thilo Albert: Software, Data curation, Validation, Visualization, Project administration 
Real-world clinical experience of extended half-life recombinant factor VIII Fc fusion protein (rFVIIIFc) in comparison to conventional factor products in patients with severe hemophilia A

Johannes Oldenburg: Supervision, Writing- Reviewing and Editing

\section{Acknowledgments}

The authors would like to acknowledge GKM Gesellschaft für Therapieforschung mbH for assistance in completion of this manuscript.

\section{Highlights}

- Real-world clinical experience of extended half-life recombinant factor VIII Fc

- Fewer injections and lower factor consumption compared to factor VIII prophylaxis

- Further optimization of adherence to prophylaxis was possible

- Annualized bleeding rates remained at least stable or slightly improved

- Perioperative hemostasis management was excellent

\section{REFERENCES}

1. Knobe, K. and E. Berntorp, Haemophilia and joint disease: pathophysiology, evaluation, and management. J Comorb, 2011. 1: p. 51-59.

2. Lobet, S., C. Hermans, and C. Lambert, Optimal management of hemophilic arthropathy and hematomas. J Blood Med, 2014. 5: p. 207-18.

3. Manco-Johnson, M.J., et al., Prophylaxis versus episodic treatment to prevent joint disease in boys with severe hemophilia. N Engl J Med, 2007. 357(6): p. 535-44.

4. Manco-Johnson, M.J., et al., Randomized, controlled, parallel-group trial of routine prophylaxis vs. on-demand treatment with sucrose-formulated recombinant factor VIII in adults with severe hemophilia A (SPINART). J Thromb Haemost, 2013. 11(6): p. 1119-27.
5. White, G.C., 2nd, et al., Definitions in hemophilia. Recommendation of the scientific subcommittee on factor VIII and factor IX of the scientific and standardization committee of the International Society on Thrombosis and Haemostasis. Thromb Haemost, 2001. 85(3): p. 560 .

6. Srivastava, A., et al., Guidelines for the management of hemophilia. Haemophilia, 2013. 19(1): p. e1-47.

7. Ingersoll, K.S. and J. Cohen, The impact of medication regimen factors on adherence to chronic treatment: a review of literature. J Behav Med, 2008. 31(3): p. 213-24.

8. Wiley, R.E., et al., From the voices of people with haemophilia A and their caregivers: Challenges with current treatment, their impact on quality of life and desired improvements in future therapies. Haemophilia, 2019. 25(3): p. 433-440.

9. Carcao, M., Changing paradigm of prophylaxis with longer acting factor concentrates. Haemophilia, 2014. 20 Suppl 4: p. 99-105.

10. European Medicines Agency (EMA), Summary of Product Characteristics: Elocta. https://www.ema.europa.eu/en/documents/ product-information/elocta-epar-productinformation_en.pdf(accessed 19 Aug 2019).

11. Peters, R.T., et al., Biochemical and functional characterization of a recombinant monomeric factor VIII-Fc fusion protein. $\mathrm{J}$ Thromb Haemost, 2013. 11(1): p. 132-41.

12. Roopenian, D.C. and S. Akilesh, FcRn: the neonatal Fc receptor comes of age. Nat Rev Immunol, 2007. 7(9): p. 715-25.

13. Powell, J.S., et al., Safety and prolonged activity of recombinant factor VIII Fc fusion protein in hemophilia A patients. Blood, 2012. 119(13): p. 3031-7. 


\section{CURRENT MEDICAL RESEARCH AND OPINION}

14. Mahlangu, J., et al., Phase 3 study of recombinant factor VIII Fc fusion protein in severe hemophilia A. Blood, 2014. 123(3): p. 317-25.

15. Young, G., et al., Recombinant factor VIII Fc fusion protein for the prevention and treatment of bleeding in children with severe hemophilia A. J Thromb Haemost, 2015. 13(6): p. 967-77.

16. Nolan, B., et al., Recombinant factor VIII Fc fusion protein for the treatment of severe haemophilia A: Final results from the ASPIRE extension study. Haemophilia, 2020. 26(3): p. 494-502.

17. Nolan, B., et al., Long-term safety and efficacy of recombinant factor VIII Fc fusion protein ( $\mathrm{rFVIIIFc)}$ in subjects with haemophilia A. Haemophilia, 2016. 22(1): p. 72-80.

18. Berntorp, E., et al., Dosing regimens, FVIII levels and estimated haemostatic protection with special focus on rFVIIIFc. Haemophilia, 2016. 22(3): p. 389-96.

19. Oldenburg, J., et al., Improved joint health in subjects with severe haemophilia A treated prophylactically with recombinant factor VIII Fc fusion protein. Haemophilia, 2018. 24(1): p. 77-84.

20. Srivastava, A., et al., WFH Guidelines for the Management of Hemophilia, 3rd edition. Haemophilia, 2020. 26 Suppl 6: p. 1-158.

21. Oldenburg, J., Optimal treatment strategies for hemophilia: achievements and limitations of current prophylactic regimens. Blood, 2015. 125(13): p. 2038-44.

22. Collins, P.W., et al., Break-through bleeding in relation to predicted factor VIII levels in patients receiving prophylactic treatment for severe hemophilia A. J Thromb Haemost, 2009. 7(3): p. 413-20.
26. Coyle, T.E., et al., Phase I study of BAY 94-9027, a PEGylated B-domain-deleted recombinant factor VIII with an extended halflife, in subjects with hemophilia A. J Thromb Haemost, 2014. 12(4): p. 488-96.

27. Giangrande, P., et al., Clinical evaluation of glycoPEGylated recombinant FVIII: Efficacy and safety in severe haemophilia A. Thromb Haemost, 2017. 117(2): p. 252-261.

28. Konkle, B.A., et al., Pegylated, fulllength, recombinant factor VIII for prophylactic and on-demand treatment of severe hemophilia A. Blood, 2015. 126(9): p. 1078-85.

29. Mullins, E.S., et al., Extended half-life pegylated, full-length recombinant factor VIII for prophylaxis in children with severe haemophilia A. Haemophilia, 2017. 23(2): p. 238-246.

30. Tiede, A., et al., Enhancing the pharmacokinetic properties of recombinant factor VIII: first-in-human trial of glycoPEGylated recombinant factor VIII in patients with hemophilia A. J Thromb Haemost, 2013. 11(4): p. 670-8.

31. Collins, P.W., et al., Recombinant longacting glycoPEGylated factor IX in hemophilia B: a multinational randomized phase 3 trial. Blood, 2014. 124(26): p. 3880-6.

32. Aledort, L., et al., A Retrospective Observational Study of Rurioctocog Alfa Pegol in Clinical Practice in the United States. J Manag Care Spec Pharm, 2020. 26(4): p. 492-503.

33. Dunn, A.L., S.P. Ahuja, and E.S. Mullins, Real-world experience with use of Antihemophilic Factor (Recombinant), PEGylated for prophylaxis in severe haemophilia A. Haemophilia, 2018. 24(3): p. e84-e92. 
Real-world clinical experience of extended half-life recombinant factor VIII Fc fusion protein (rFVIIIFc) in comparison to conventional factor products in patients with severe hemophilia A )

34. Peyvandi, F., et al., Real-life experience in switching to new extended half-life products at European haemophilia centres. Haemophilia, 2019. 25(6): p. 946-952.

35. Pouplard, C., et al., Multicentre pharmacokinetic evaluation of rFVIII-Fc (efmoroctocog alfa) in a real life and comparison with non-extended half-life FVIII concentrates. Haemophilia, 2020. 26(2): p. 282-289.

36. Simpson, M.L., et al., Comparing Factor Use and Bleed Rates in U.S. Hemophilia A Patients Receiving Prophylaxis with 3 Different Long-Acting Recombinant Factor VIII Products. J Manag Care Spec Pharm, 2020. 26(4): p. 504-512.

37. Tagliaferri, A., et al., Optimising prophylaxis outcomes and costs in haemophilia patients switching to recombinant FVIII-Fc: a single-centre real-world experience. Blood Transfus, 2020. 18(5): p. 374-385.

38. Holmström, M., et al. Real-World Usage of rFVIIIFc in Sweden: A Report from the Swedish National Registry for Bleeding Disorders. Presented at the 13th Annual Congress of the European Association for Haemophilia and Allied Disorders (EAHAD); 5-7 February 2020, The Hague, Netherlands. 2020 .

39. Oldenburg, J., et al. No Relapse in Patients with Previous Inhibitors Switched to rFVIIIFc in Ongoing Observational Phase 4 Studies. Presented at the International Society on Thrombosis and Haemostasis (ISTH) 2020 Virtual Congress; 12-14 July 2020. 2020.

40. Keepanasseril, A., et al., Switching to extended half-life products in Canada preliminary data. Haemophilia, 2017. 23(4): p. e365-e367.
41. Iorio, A., et al., Indirect comparisons of efficacy and weekly factor consumption during continuous prophylaxis with recombinant factor VIII $\mathrm{Fc}$ fusion protein and conventional recombinant factor VIII products. Haemophilia, 2017. 23(3): p. 408-416.

42. Klamroth, R., et al., Rurioctocog alfa pegol PK-guided prophylaxis in hemophilia A: Results from the phase 3 PROPEL study. Blood, 2020.

43. Peyvandi, F., et al., Kreuth V initiative: European consensus proposals for treatment of hemophilia using standard products, extended half-life coagulation factor concentrates and non-replacement therapies. Haematologica, 2020. 105(8): p. 2038-2043.

44. Habermann, B., et al. S2k-Leitlinie Synovitis bei Hämophilie. 2018.

45. Federal Medical Association. CrossSectional Guidelines for Therapy with Blood Products and Plasma Derivates. 5th revised and updated edition 2020. Pages 118-146

46. Ar, M.C., et al., Methods for individualising factor VIII dosing in prophylaxis. Eur J Haematol Suppl, 2014. 76: p. 16-20.

How to cite this article: Goldmann G., Marquardt N., Horneff S., Klein C., Albert T., Oldenburg J. Real-world clinical experience of extended half-life recombinant factor VIII Fc fusion protein (rFVIIIFc) in comparison to conventional factor products in patients with severe hemophilia A. Journal of Current Medical Research and Opinion. 2021;950-960. https://doi.or g/10.15520/jemro.v4i05.422 


$\begin{array}{ll}\text { Abbreviations } \\ \text { ABR } & \text { annualized bleeding rate } \\ \text { AjBR } & \text { annualized joint bleeding rate } \\ \text { AsBR } & \text { annualized spontaneous bleeding rate } \\ \text { AsjBR } & \text { annualized spontaneous joint bleeding rate } \\ \text { AtBR } & \text { annualized traumatic bleeding rate } \\ \text { EHL } & \text { extended half-life } \\ \text { FVIII } & \text { factor VIII } \\ \text { HCV } & \text { hepatitis C virus } \\ \text { HEK } & \text { human embryonic kidney } \\ \text { HIV } & \text { human immunodeficiency virus } \\ \text { Ig } & \text { immunoglobulin } \\ \text { IU } & \text { international unit } \\ \text { PwHA } & \text { patients with hemophilia A } \\ \text { rFVIII } & \text { recombinant factor VIII } \\ \text { rFVIIIFc } & \text { recombinant factor VIII Fc fusion protein, efmoroctocog alfa } \\ \text { SHL } & \text { standard half-life }\end{array}$

